\title{
IOT Based Portable Medical Kit
}

\author{
Kartik Arora, Sanjay Kumar Singh
}

\begin{abstract}
Medication requirements and health issues have been on the rise in the last decade and to cater to this numerous technologies have been introduced to the health sector. Internet of Medical Things (IoMT) has been on the rise and providing medical care to the much needed through services, smart medical devices that not only provides an enhanced platform to the doctor but also to the patients to maintain better healthcare levels. This paper addresses the implementation of a multilayered architecture for a health monitoring kit incorporated with sensors and alert mechanism to activate a pill dispenser. The sensor data is relayed to a personalized android application and care has been taken to make sure that the patient receives the medicine on time and in the required dosage.
\end{abstract}

Index Terms: Android application, Dispenser, Health sensors Medicine, Monitor

\section{INTRODUCTION}

These days global ageing and the prevalence of chronic diseases have become a major concern. The evolving technologies have led to a rise in the life expectancy of the elderly people which will contribute to the $22 \%$ of the population by 2050 as predicted by the United Nations. One of the most evolving technologies in medical field, the Body Sensor Network (BSN) that are light weighted and are used to monitor human body functions and the surroundings. [1] The medical researchers suggest about $80 \%$ of the aged people suffer from at least one chronic disease. A drastic shift is being made by many countries to change their hospitals by decreasing the number of patient beds and increasing the proportion of home healthcare to provide medication from home. Shifting healthcare from the hospitals to households is becoming a promising trend. This has multiple benefits, firstly people can be giving all the medical facilities in the comfort of their home. Second, this reduces the financial burden due to the massive hospital bills. Third, all the hospital resources can be dispatched at the time of need and as per requirement.

A shift from home healthcare system and services can reduce the major medical expenditure and enduring an alternative treatment by providing a healthy lifestyle through routine self-checkups. The use of intelligent components, Radio Frequency Identification (RFID) tags, embedded sensors and actuators etc. with the help of latest technology has set in the

Revised Version Manuscript Received on Jun 20, 2019.

Kartik Arora, Electronics and Communication Engineering (UG), Vellore Institute of Technology, Vellore, India

Sanjay Kumar Singh, School of Electronics Engineering Vellore Institute of Technology, Vellore, India trend for Internet of Things (IoT) which is being rapidly developed and significantly expanding its scope, therefore booming the number of IoT-based applications in the market. The wireless connectivity through Bluetooth or Wi-Fi can be used to send data through different transmission media like $3 \mathrm{G} / 4 \mathrm{G}$ or Wi-Fi.[2] The growth of these technologies have led to the integration of IoT devices in many homes for $24 / 7$ healthcare as well as comfort purposes. The lasts trend has not only led to smart systems but also wearable devices and systems in IoT scenario to achieve better care of the patient and e-health services. Mobile-phones provide easy telemonitoring system for the smart systems and the wearable devices.

A new mean to measure non-invasive bio-signal measurement, smart health monitoring chair has been introduced lately. But the solution to these are exclusive and therefore implemented using off-the-peg components which leads us to a conclusion that their physical size, rigid nature and short battery exerts limits for its potential long-term use. Nowadays patient's utmost priority is safe and high-quality healthcare service leading to healthcare data security and patients' privacy as the most important issues that will have a great impact on the future growth of IoT in healthcare [3]. Privacy of the user is of major concern in IoT based healthcare systems. Usually a healthcare device shares data with the authorized devices and all the trusted clinics and professionals. This data may also be shared with hospitals and pharmacies and some health insurance firms. The loss of user data is prevailing in recent times, patient data can be vulnerable to hackers during cloud transfer or synchronization with other nearby coordinated devices. Hence, there is a need to protect this information from unauthorized access by hackers that may lead in the posting of personal information in the public domain or it may interfere with essential nearby medical equipment like a pacemaker. A security threat to the user's monitoring devices and data may make the patient face social embarrassment, mental disorders, or adverse physical effects such as a fatal heart attack. Hence data security and personnel authorization is extremely important in healthcare monitoring using IoT. The concept of Internet of Medical Things (IoMT) emerges as an evolving technology that may provide an advanced remote healthcare and telephone health services with the help of IoT and its applications. It guarantees various issues like time efficiency, management costs, patient experience and feedback.[4]

\section{LITERATURE SURVEY}

The number of devices for medication administration for non-professional users is on a rise. They are either manual, with






\section{IOT Based Portable Medical Kit}

multiple boxes for pills just like the design by Philips or has a single box which is divided in to a number of cavities that can be filled with medicines.

Each cavity can hold different sizes as well as a different set of medicines. The user is required to update the medicine in each tray as per his requirements for the required number of days. The existing devices do not provide any alarm to indicate the time of taking the medicine. A number of medicine reminders in the market like a pill "Pill Mate" is a gadget that provides both audio and visual signals to the user to remind him/her through a buzzer and a display. It reminds at a pre-set time, usually set by the user or his/her guardian to take medicines timely. A smart phone application could be designed to help patients/users to avoid mistakes as a lot of elderly people are aware of the android applications and are now habitual to use those. It reminds its users to take correct medicines on time and record the refill schedule for later review by healthcare professionals or personal doctors.[10-1,2] $74 \%$ of total death count in the hospital is caused due to overdose or under dosage of the medicines as revealed by The Patient's Safety Authority of India (PSAI).[11] Further 5 year report from 2004 to 2008 mentions about the medication errors resulting from various breakdowns in the process of obtaining, documenting, and/or communicating patient weights that often leads to deaths of the patient or wrong medication. The various statistics state that out of the 479 reports, $93.5 \%$ reports represent the five most common medication error event types, with the most commonly reported event type being wrong dose/over dosage that accounts up to $43.4 \%$ and wrong dose/under dosage that holds up to $21.3 \%$, which holds up a huge number in medication error [11-2].

\section{ARCHITECTURE}

The device is a multi-layered structure as shown in the figure. The architecture consists of 3 layers: sensor network, dispenser, Integrated Application.

\begin{tabular}{|c|}
\hline \multicolumn{2}{|c|}{ Integrated Application } \\
\hline Data Collection \\
\hline Network And Gateway (Dispenser and Sensors) \\
\hline Arduino UNO Arduino Mega2560 \\
\hline Pluetooth \\
\hline Fig 1. Archictecture Flow
\end{tabular}

The hardware components provide the dispenser controller with two kinds of facilities, first, hardware control which controls the commands that the controller requests, second, event notification which is the primary means of communication from hardware to controller and vice versa. The controller commands the alert module as well as the dispenser in order to make the system functional.

The prototype uses a microcontroller which activates the GSM module to notify the user for the medication based on a pre- decided time. Simultaneously it activates the pill dispenser for short period of time to dispense a pill. The dispenser to avoid any sort of misinterpretation is mounted on a height of 66 inches from the ground.

\section{A. Pill Dispenser}

The dispenser involves the use of an ultrasonic sensor depending on which the dispenser dispenses pills to the patient. The sensor is active for a certain period of time after the alert message has been sent to the user. This allows to prevent dispensing the pill to the wrong person as the patient will be time bound to take his/her medicine. Further the dispenser also makes sure that no toddler or a physical device present in the room is able to deceive the sensor for Bing a hand, this is made possible by situating the dispenser at a formidable height from the ground and controlling the range of the sensor. The dispenser keeps a track of the amount of pills left in stock and displays it on an LCD. This is governed by initially setting the number of pills placed in the dispenser and at each activation period.

\section{B. Sensor network}

The sensor network is the central layer and the origin of information collection of the health monitoring system. This layer collects the data which is clustered up to be transmitted to the next layer and is like the front end of any website.

\section{Sensor interface}

Various number of health sensors are employed to collect the patient's essentials and monitor his or her surroundings. The sensor interface consists of multiple contact sensors that need to be calibrated well as these sensors are of high importance to the medical industry and medical professionals and are used to assess health care consistently.

\section{Pulse sensor}

This sensor is responsible to monitor the pulse of the patient. The pulse if the number of times the human heart beats in one minute. It is based on the principles of optoelectronics. The sensor includes 2 sides, on one side we have an LED alongside an ambient light sensor and on the other side the circuit responsible for noise cancellation and amplification.

\section{E. Heart rate sensor}

this is a more sophisticated version of the pulse sensor which measures the heart rate by using an LED and a phototransistor. The phototransistor is responsible for detecting the flux change when a finger is placed in between the LED and the photo transistor.

\section{F. ECG sensor}

this sensor is responsible to measure the heart's electrical activity. This can be stated as the electrocardiogram. This is important to understand the physiological, emotional arousal. Multiple electrodes are used to detect the electrical activity. These electrodes are attached to the human body to collect reliable and assertive readings.

\section{G.Accelerometer}

this device measures the static acceleration due to gravity for tilt scene applications as well as dynamic acceleration in cases of

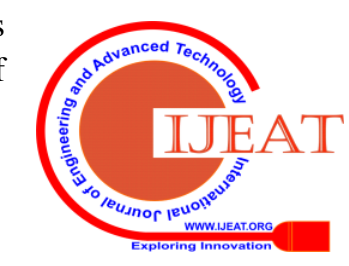


shocks and fall. This sensor can also be used to detect if the object is in motion or in the state of rest. The sensors uses piezoelectric elements which when come in contact with a round free moveable object inside the sensor produce electric current.

\section{H.Body temperature}

the sensor when brought in contact with the skin can detect the body temperature and thus any abnormality related to temperature like fever or hypothermia can be detected. These sensors provide an accurate reading and can be used to replace the traditional thermometers.

\section{Alert Module}

The alert module is also an android application. This enables us to alert the patient via notification.

\section{J. Integrated Application}

The integrated application is an android application that receives data from the Arduino that mounts all the health sensors. When these sensors are activated, the read data is sent to the android application that can be reviewed by the doctor and prescribe medicines accordingly.

\section{RELATED WORKS}

There is an out sized variety of medication administration help devices for users. Most complete devices (e.g., [3]-[5]) accessible these days are manual, the user needs to load the device with the prescribed medication and reset the system after each use, this is a major improvement in our pill dispenser.

Like schedules employed in our dispenser, medication schedules manoeuvred by automatic devices and planning tools like the, "MEDICATE" Tele-assistance System [6], [7] and "Magic Medicine Cabinet" [8] can be regulated to atone for user timing and condition changes. Comparably, our dispenser could be a complete tool, and is capable of adjusting the schedule as per the necessities of the patient or a modification in prescriptions. The dispenser is designed for individuals who do not need constant monitoring and supervision but instead need a device to track their pill cycle during the course of the day. The device is a supplement for all the reminders on paper or our mobile phones that constantly nag the user with notifications, not just the notifications the user is now free from keeping a track of all his pill refill, instead the device keeps a track for the user and reminds him when refill is required.

This section discusses the literature of IoT based remote healthcare systems. A healthcare system most commonly seen in hospital is proposed in [3]. This incorporates a node attached to the patient's body which transmits the health status of a patient to a central monitoring system. Shu-Yuan Ge et al. in his paper show cases a design that is primarily the integration of 11073 IEEE Service/DIM and CoAP to try on devices of medication and healthcare so they can be used in IoT system and its application. They have also carried out a performance comparison between Both HTTP nad11073 DIMwith the help of CoAP and other technologies. It also asses the performance with CoAP and HTTP in regard to the packets abundance in single transaction, data loss rate and syntax by the use of JSON and XML. As a conclusion CoAP transmitted lesser packets as compared to HTTP. Further they concluded that XML is not better than JASON in terms of resource consumption. [5-7].Iuliana Chiuchisan et al. presents an intelligent system for the Parkinson's infection test. His proposed system not only supports but also assists the doctors in the medical treatment, diagnosis, prescription and overall progress. [5-11].

Georges Matar et al. comes up with a technique that helps us to monitor the patient's posture by using his or her body weight which is applied on specially designed mattress and then the measured pressure is used to monitor the patient's posture. His work has been ensured and verified by Cohen's Coefficient whose value is 0.866 which concludes high accuracy of detection. He claims the purpose of his work is to reduce the cost. [5-8].

In [3] the authors propose UDA-IoT system which is used in case of emergency. This system is responsible to collect, integrate and interoperate data in various medical applications and forward it for monitoring or processing.

Kuo-hui yeh et al. the advancement in the communication brings new era of IoT which is based on network and wireless communication like the Samsung's smart home using Artificial Intelligence. The author proposes an IoT based system that uses body sensor networks as the ground work for efficiency and robustness especially for public networks based on IoT. The system is kept secured by proposing a security parameter. The author guarantees the proposed system and scheme that it is more suitable to apply the scheme to the common mobile object [5-38]. Granados et al. [6-14] proposes web enabled gateways for health cased Iot systems. As the wired gateways provide high efficiency and lower cost, the author installed wired gateways in areas of restricted movement. Radio frequency identification (RFID)-based eHealthcare systems are proposed in [6-15, 16] where the authors design a system to monitor the patients environmental conditions that is if the surroundings the patient belong to like temperature, humidity with the help of respective sensors, then this data is transmitted to the cloud for a more comprehensive study of the data.Catar- inucci et al. [6-16] proposes a smart system based architecture to monitor a patient's medical condition by using an ultra-high frequency Radio frequency identification.

\section{IMPLEMENTATION}

\section{A. Monitoring system:}

The sensors are connected to the microcontroller, which governs the working of the sensors. Each sensor has a specific role to perform hence each and every sensor collects data. Temperature, electrocardiogram, pulse, body orientation, heart rate are monitored and these readings are relayed to the customized android app so that this data can be made visible to the authorized personnel.

\section{B. Dispenser:}


The user when dealing with the dispenser has the tools to adjust the dispenser's schedule according to their requirements or their prescriptions. The user programs his prescription details in the microcontroller i.e. specifying the number of pills to be used, the timings of the day when the dispenser is supposed to alert the user regarding his medication. On entering the essential details the user is free to handle his/her daily chores and need not be bothered about his medication routine or the dosage requirements. The dispenser will be activated at regular intervals and the medicines will be dispensed depending on the presence of the user. The dispenser is designed for individuals who do not need constant monitoring and supervision but instead need a device to track their pill cycle during the course of the day. The device is a supplement for all the reminders on paper or our mobile phones that constantly nag the user with notifications, not just the notifications the user is now free from keeping a track of all his pill refill, instead the device keeps a track for the user and reminds him when refill is required.

\section{Data collection}

While the sensors are supposed to interact with the outside world their values need to be collected, aggregated and

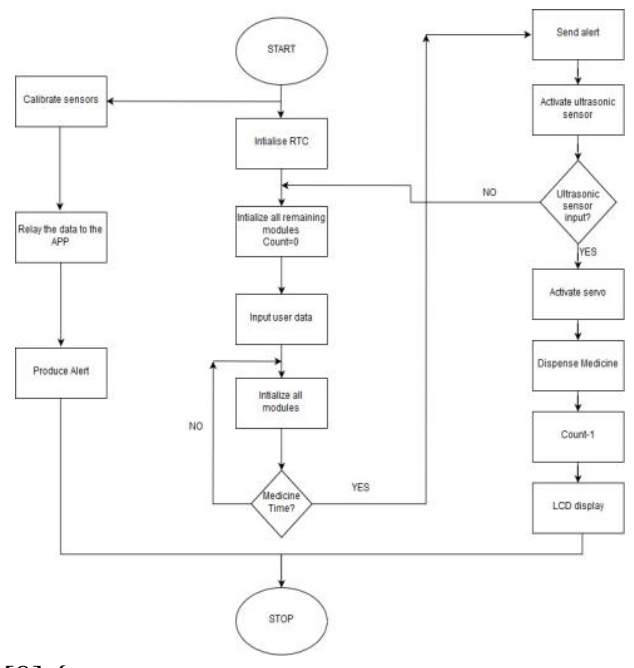

[9] \{

[10] Rotate Servo

$[11]\}$

[12]\}

[13]\}

[14] Count=count -1

[15] Repeat steps 3 to 14

[16] END

\section{Sensor Module}

Input: User sensor input

Output: Sensor analog output

[1] Find all connected sensors

[2] For (user==valid)

[3] \{

[4] Read analog data

[5]\}

[6] Repeat till sensor value==NULL

[7] Process Sensor value

[8] Activate Bluetooth

[9] Transmit all analog values parsed. Microcontrollers are used in this sub-layer. The microcontroller collects the data from its attached sensors processes, parses it. This data is made ready to be wirelessly transmitted to the further layers. Microcontrollers use analog to digital converters to convert the physical analog readings into digital form. 2 different microcontrollers are used: one in sensor interfacing and one in pill dispensing and the two microcontrollers are kept in sync using Bluetooth.

\section{Clock synchronization}

The real time clock is responsible for the complete functioning of the dispensing system. The clock works on a crystal oscillator and is responsible for accurate time keeping. The clock makes sure that the dispenser is activated on time and the dispensing system is able to complete the function Not just this the cock keeps a track of the number of Pills dispensed the time it was dispensed and the next cycle for dispensing the pill.

\section{ALGORITHM}

Dispenser Module

[1] Calibrate RTC from PC

[2] Pills Count=3

[3] For (RFID==valid)

[4] \{

[5] If (medicine time)

[6]\{

[7] Read ultrasonic sensor distance

[8] If $(10<$ distance $<15)$

[10] END

\section{RESULTS AND DISCUSSION}

The figures below show the actual output received and transmitted to the integrated application. The ECG graph displays real time values with time along the $\mathrm{x}$-axis and signal/mV along the $y$-axis.

All the sensor readings fall in the normal range of the expected values.

(2) сомб

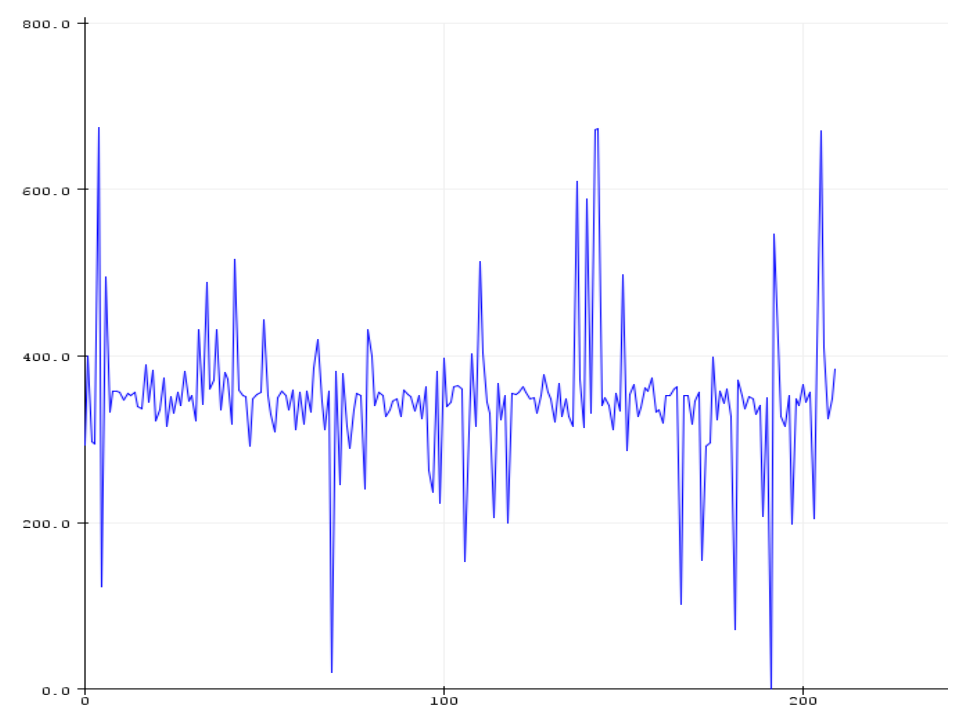

Fig 3. ECG Output

The dispenser module hardware works perfectly with the result as shown in the figures below. The pill count decreases as 
expected and the message "Medicine Time" is displayed on the appropriate set time by the user.
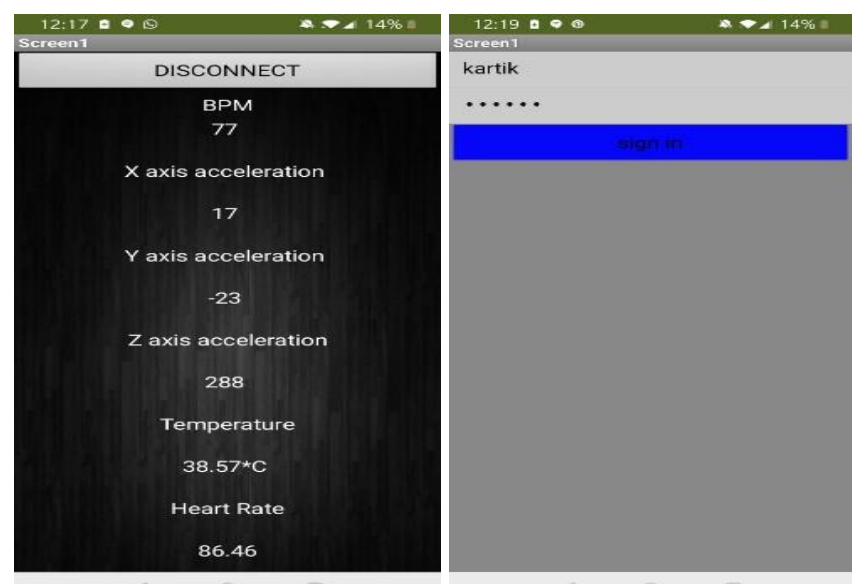

Fig 4. Log in Page and Sensor output Values of Application

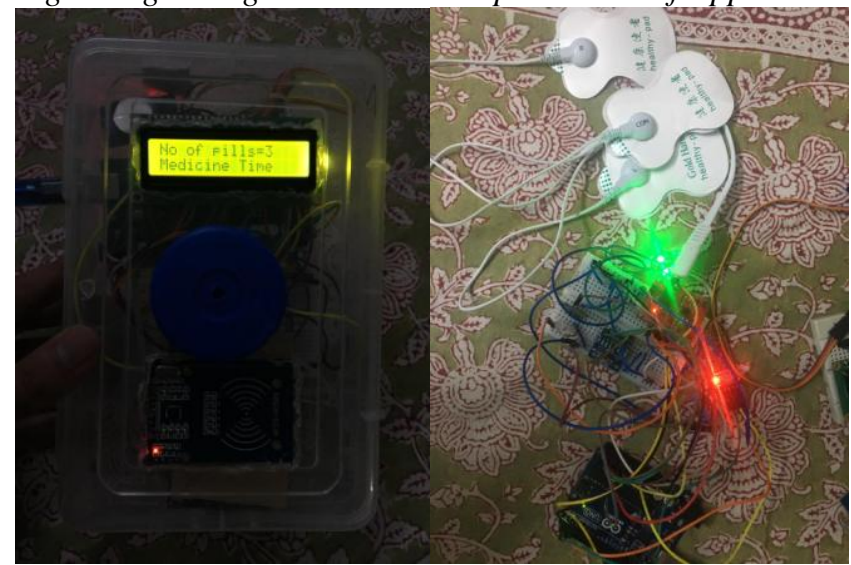

Fig 5. Hardware Implementation

\section{CONCLUSION}

The pill dispenser provides a means of regular medication at predefined time intervals. The dispenser makes sure that the user is alerted every time he/she needs to take a dose, this way the user need not worry about setting reminders for his daily medicine schedule.

The dispensing mechanism is designed to make sure that no stray objects can cause the pill to leave the dispenser thus making the system less vulnerable. More over the automated system makes sure that the right amount of dosage is being consumed by the user and this rules out the possibility of over and under dosage.

The integrated health sensors with the right range of values provides a mean of routine health checkup from home without the hassles of visiting doctors where the doctor can provide medication by analyzing the data sent through the integrated application or call the patient to clinic if it's something serious

\section{ACKNOWLEDGMENT}

The idea of making a portable medical kit was successfully implemented under the guidance of Professor Sanjay Kumar Singh at Vellore Institute of Technology, Vellore. I would also like to thank the Head of Department, Prof.
Thanikaiselan V. for providing me a platform to implement my idea.

\section{REFERENCES}

1. Prosanta Gope and Tzonelih Hwang, "BSN-Care: A Secure IoT-Based Modern HealthcareSystem Using Body Sensor Network" 1368IEEE SENSORS JOURNAL, VOL. 16, NO. 5, MARCH 1, 2016

2. Kaleem Ullah,Munam Ali Shah,Sijing Zhang,"Effective Ways to Use Internet of Things in the Field of Medical and Smart Health Care" 978-1-4673-8753-8/16/\$31.00 @2016 IEEE

3. Punit Gupta, Deepika Ahrawal“'IoT Based Smart Health Care Kit”. 2016 ICCTICT

4. Abdelrahman Rashed, Ahmed Ibrahim, Ahmed Adel, Bishoy Mourad, Ayman Hatem, Mostafa Magdy,Nada Elgaml, Ahmed Khattab "Integrated IoT Medical Platform for RemoteHealthcare and Assisted Living". 978-1-5386-1359-7\$31.00c@2017 IEEE

5. Gulraiz J. Joyia, Rao M. Liaqat, Aftab Farooq, and Saad Rehman"Internet of Medical Things (IOMT): Applications, Benefits and Future Challenges in Healthcare Domain"

6. M.Shamim Hossaina,Ghulam Muhammad"Cloud assisted Industrial Internet of Things (IIoT) - Enabled framework for health monitoring”

7. "Combination of Cloud Computing and Internet of Things (IOT) in Medical Monitoring Systems"

8. D. Wan, "Magic medicaine cabinet: A situated portal for consumer healthcare," in Proc. First Int. Symp. Handheld and Ubiquitous Com-puting (HUC '99), Sep. 1999

9. Kuperman GJ, Bobb A, Payne TH, et al. "Medication Related Clinical Decision Support in Computerized Provider Order Entry Systems: A Review" Journal of American Medical Informatics Association, 2007.

10. S. Mukund1 and N.K.Srinath "Design of Automatic Medication Dispenser"

11. “Automatic Pill Dispenser”, Mrityunjaya D H1, Kartik J Uttarkar2, Teja B3, KotreshHiremathInternational Journal of Advanced Research in Computer and Communication Engineering CertifiedVol. 5, Issue 7, July2016

\section{Authors ProfiLe}

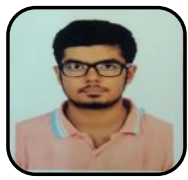

Kartik Arora pursing B.Tech, final year, in Electronics and Communication Engineering from Vellore Institute of Technology, Vellore. A passionate student with interest in IoT and embedded field planning to pursue future in research.

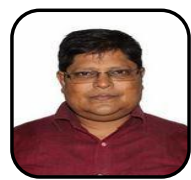

Dr. Sanjay Kumar Singh, Associate Professor in Department of Electronics and Communication Engineering at Vellore Institute of Technology, Vellore. 